\title{
Solvatochromic Dyes as pH-Independent Indicators for I onophore Nanosphere-Based Complexometric Titrations
}

\author{
Jingying Zhai, Xiaojiang $X i e$ and Eric Bakker \\ Department of Inorganic and A nalytical Chemistry, U niversity of Geneva, Quai Ernest-A nsermet \\ 30, $\mathrm{CH}-1211$ Geneva, Switzerland \\ Email: eric.bakker@unige.ch
}

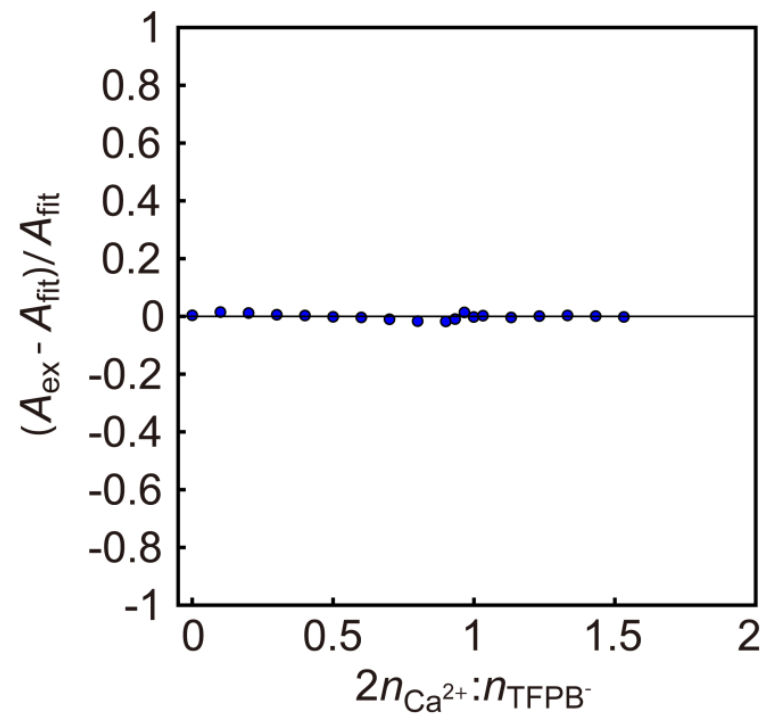

Figure S1. An estimation of the error between theoretical fit and the experimental data for Figure $2 b$. $A_{\text {ex }}$ is the experimental absorbance at $586 \mathrm{~nm}$ and $A_{\text {fit }}$ is the corresponding theoretical absorbance used to fit the data. 

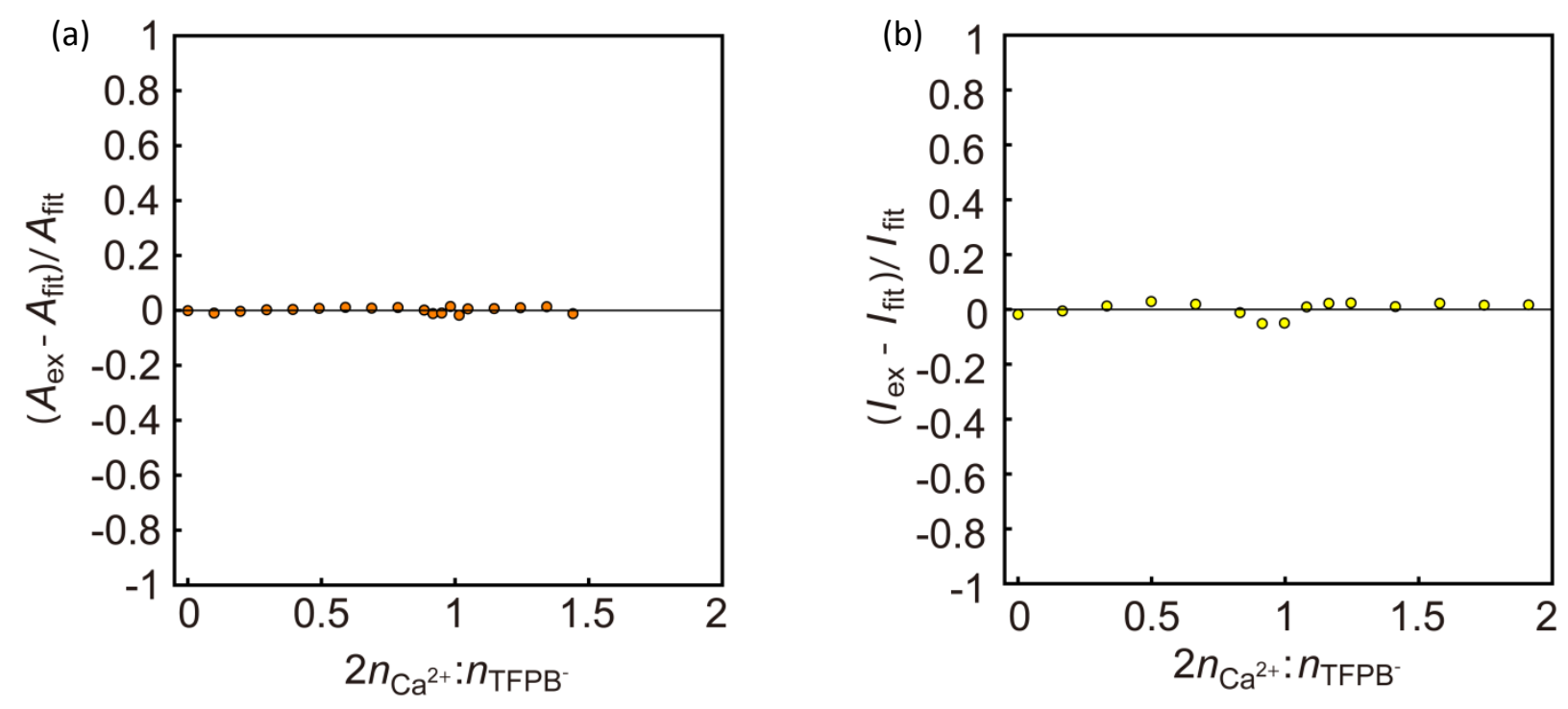

Figure S2. An estimation of the error between theoretical fit and the experimental data for Figure $3 a$ (left, a) and Figure 3b, (right, b). (a) $A_{\text {ex }}$ is the experimental absorbance at $586 \mathrm{~nm}$ and $A_{\text {fit }}$ is the corresponding theoretical absorbance used to fit the data. (b) $\mathrm{I}_{\mathrm{ex}}$ is the experimental maximum emission intensity and $\mathrm{I}_{\mathrm{fit}}$ is the corresponding theoretical intensity used to fit the data.

Table S1. Comparision between the complexometric titration methods based on ion selective nanospheres and water soluble reagents.

\begin{tabular}{ccc}
\hline & Nanosphere based & W ater soluble reagents \\
\hline Selectivity & high & low \\
Variety of the chelators and indicators & large & small \\
W orking pH range & wide & narrow \\
C ost & high & low \\
\hline
\end{tabular}

